\title{
An introduction to the Current awareness section
}

\author{
W. R. LEE AND K. S. WILLIAMSON
}

This new section in the Journal is a classified list of references with key words likely to be useful to readers. The great increase in world scientific literature during the past 20 years is causing concern in many branches of medicine. It is becoming progressively difficult for the worker in a specialized field to become aware of all relevant publications. In occupational medicine the situation is particularly difficult, for not only are relevant papers published in the specialist journals, but a number of important papers appear in general medical publications and some in journals devoted to other fields such as dermatology and chest diseases. Although papers on specific topics may be traced through indexing journals, these may not be readily accessible, and at present there is no means of scanning the overall output of recent relevant papers so that the reader may keep abreast of developments in the field. It is in an attempt to remedy this deficiency that we have explored the use of computer reference retrieval systems and are using one such system to compile a list of recent papers about occupational health to form the new Current awareness section which is introduced in this issue. The source of this list is the MEDLARS information retrieval service. It is possible that other information retrieval services could be used in the future to improve the coverage, but it seems likely that MEDLARS will remain the principal source of information.

MEDLARS denotes Medical Literature Analysis and Retrieval System. The system was developed by the National Library of Medicine (N.L.M.) in the United States and came into operation in 1964. The N.L.M. collects papers from all the major medical journals published throughout the world (about 2,800), and these constitute the input to the MEDLARS system. They are examined by a team of indexers, who allot to each paper a number of index terms describing the essential concepts contained in the paper. The number of indexing terms used to describe a paper varies with the number of concepts - covered by that paper and averages about 10 . Index terms are selected from a controlled list known as MeSH (medical subject headings). MeSH terminology, though open to extension and revision, is rigidly controlled, any concept being represented by only one word or phrase out of the possible grammatical variations and synonyms. The current MeSH is published each January as part of Index Medicus.

The indexers arrange information about title, authors, journal reference, and indexing terms of each paper and this information is then recorded on magnetic tape. Every month a new tape is added to the series. The centrally produced magnetic tapes are copied and thus made available in other locations where there is a suitable computer (e.g. in the United Kingdom).

Over the past 12 months we have evolved a formulation in MeSH terms of the field of occupational health. This programme will be used for regular searching of the newly received MEDLARS tapes. The references printed out are reviewed and irrelevant ones discarded. The remainder are arranged in a classification described later. The entry for each reference will consist of the title (translated into English if necessary), a note of the original language, the authors, the journal reference, and also the list of indexing terms applied to the paper. Although abstracts will not be produced, the indexing terms should provide a considerably better basis for readers' selection of relevant papers than titles alone.

The references will be classified into one of the 11 main classes shown in the Table. No attempt will be made to order the papers within each class but, as the title of the paper will be printed first, identification of references of interest to the reader should be quite rapid. Each reference will be printed once only, so that those papers which could be entered into more than one class will appear only in the section which seems to be most appropriate.

The delay between publication of a paper in its source journal and its appearance in our Current 
awareness section will vary, depending on how soon the reference enters the U.K. MEDLARS system, and the time needed to convert the computer print-out into a form suitable for publication. We hope that future developments in the U.K. MEDLARS system, including perhaps computercontrolled printing, will reduce any delay between a paper entering MEDLARS and its appearance in the British Journal of Industrial Medicine.

The performance of the MEDLARS search programme has been tested in a number of ways. The search programme retrieved more than $75 \%$ of the papers published in a group of specialist journals; its performance in retrieval of papers from nonspecialist journals cannot be accurately assessed by such a simple method because of the very large number of periodicals involved. The programme's main defect in its present form is that it fails to retrieve a proportion of papers dealing with industrial toxicology, particularly those papers which are concerned with the laboratory or theoretical aspects of the subject. We hope to find ways of modifying the search programme in the future, so that its performance in the field of toxicology will be improved. Additionally, pilot investigations are being made on other information retrieval systems to examine their ability to produce relevant information in industrial toxicology.

This use of a computer-based information retrieval system may be important and the technique may well have applications in other fields of medicine. It involves the use of costly and complex facilities and it is necessary to establish whether the Current Awareness section provides a useful service to readers of the journal. We propose to ask for opinions by means of a questionnaire, which will be circulated when readers have had time to assess the value of the section.

This project has been supported by financial grants from the British Medical Journal and from the Office for Scientific and Technical Information of the Department of Education and Science.

We should like to thank Dr. J. G. Pusey of the Office for Scientific and Technical Information for his continued advice and help at all stages of this project, also Mr. Glyn Evans, formerly MEDLARS liaison officer at the Royal Society of Medicine, and Dr. A. J. Harley of the National Lending Library, for their advice and help in preparing the search programme. We are grateful to Dr. Martin Ware and Mr. C. G. A. Parker, the Editor and the General Manager of the British Medical Journal, for their interest and help.

\section{TABLE}

\section{Classification of Papers}

(1) Historical, legislative, and general

(2) Organization of medical and nursing services and education

(3) Physiological, psychological, and organizational aspects of work and rehabilitation

(4) Traumatic conditions, accidents, and accident prevention

(5) Effects of inorganic substances and minerals

(6) Effects of organic substances

(7) Effects of animal and vegetable substances

(8) Effects of physical agents

(9) Methods of investigation of body function

(10) Environmental measurement, control, and protection of the subject

(11) Miscellaneous 